\title{
Geopolíticas del Conocimiento Psicológico y Riesgos Psicosociales: Reproduciendo la Subordinación por Medio de las Tesis de Grado y Postgrado
}

\section{Geopolitics of Psychological Knowledge and Psychosocial Risk: Reproducing Subordination by Means of Graduate and Undergraduate Theses}

\author{
Hernán Camilo Pulido-Martínez y Ana María Urbina-Barón \\ Pontificia Universidad Javeriana
}

\begin{abstract}
En este artículo se presentan los resultados de un estudio que indagó por las relaciones geopolíticas de la psicología. Se examinaron 108 tesis de pregrado y postgrado, realizadas en el área de riesgos psicosociales en 7 de las principales universidades de Bogotá, Colombia, entre 1991 y 2014. Se analizaron los siguientes aspectos: los años de presentación de las tesis, la institución académica que las acogió, el programa específico al que estaban vinculadas, el título asignado a la investigación y el diseño utilizado. También se establecieron las referencias consultadas, el idioma en el cual estas se habían publicado y el tipo de autoría que estas presentaban: nacional, internacional, investigadores particulares e instituciones reguladoras del trabajo. Los resultados señalan los canales a través de los cuales circula el conocimiento psicológico sobre los riesgos psicosociales, el desvanecimiento de las fronteras disciplinares de la psicología, así como la emulación que ocurre en la construcción local de la psicología y su consecuente subordinación a las redes internacionales del conocimiento.
\end{abstract}

Palabras clave: riesgos psicosociales, geopolítica, tesis, psicologización

\begin{abstract}
This article presents the results of a study on the geopolitical relationships of psychology. One hundred and eight undergraduate and graduate theses on the topic of psychosocial risks were examined. They were produced by students of 7 of the main universities of Bogotá, Colombia, between 1991 and 2014. The following aspects were analyzed: the years when the theses were submitted, the academic institution where they were produced, the specific program to which they belonged, the title assigned to each research project, and the design used. In addition, the present study also identified the references consulted, the language in which they had been published, and their type of authorship: national, international, independent researchers, and labor regulation agencies. Results reveal the channels through which psychological knowledge about psychosocial risks circulates, the fading of disciplinary boundaries of psychology, and the emulation that takes place in the local construction of psychology, as well as its consequent subordination to international knowledge networks.
\end{abstract}

Keywords: psychosocial risk, geopolitics of psychology, theses, psychologization

Desde hace ya varias décadas se ha venido señalando en la literatura la urgente necesidad de estudiar aspectos sociales, culturales y económicos que están involucrados en la expansión del conocimiento psicológico alrededor del mundo (Brock, 2006; Castaño \& Sánchez, 1978; Herman, 1995). Aun así, muy pocos investigadores se han concentrado en estudiar la geopolítica del conocimiento psicológico, entendida esta en términos de las relaciones asimétricas de poder que se derivan del hecho de que la mayoría de los objetos psicológicos se producen en los países del Atlántico norte, Estados Unidos, Reino Unido, Alemania y Francia (Brock, 2006; Danziger, 1997; Molinari, 2004), como de la situación, fácilmente constatable, de que desde esos lugares dichos objetos migran para ser adaptados, replicados e hibridados para su uso local en diferentes ámbitos institucionales alrededor del mundo (Blowers \& Turtle, 1987; Moghaddam, 1987; Pulido-Martínez \& Carvajal-Marín, 2016). En otras palabras, la geopolítica de la psicología se ha dejado de lado en la investigación, especialmente en los países de la llamada "periferia", en donde este conocimiento y sus prácticas derivadas se consumen como productos importados (Staeuble, 2006).

Hernán Camilo Pulido-Martínez y Ana María Urbina-Barón, Facultad de Psicología, Pontificia Universidad Javeriana, Bogotá, Colombia.

La correspondencia relativa a este artículo debe ser dirigida a Hernán Camilo Pulido-Martínez, Facultad de Psicología, Pontificia Universidad Javeriana, Carrera 7 N 40-00 Edificio Manuel Briceño, oficina 203, Bogotá, Colombia. E-mail: cpulido@javeriana.edu.co 
PULIDO-MARTÍNEZ Y URBINA-BARÓN

Con miras a contribuir al incipiente debate sobre la geopolítica del conocimiento psicológico, se presenta en este artículo un análisis de las tesis de pregrado y postgrado realizadas entre los años 1991 y 2014 en relación con los riesgos psicosociales en el trabajo en siete de las principales universidades localizadas en Bogotá, Colombia. El estudio se propuso realizar un análisis de corte bibliométrico que estableció: la producción de tesis en las universidades consideradas, las facultades que acogieron los estudios y los temas principales que se investigaron. Asimismo, se realizó un recuento de las fuentes conceptuales utilizadas y se establecieron sus lugares de origen.

\section{Geopolítica del Conocimiento y Psicología}

Tradicionalmente, los estudios que se han encargado de estudiar la expansión de la psicología proponen recuentos acerca del estado de la disciplina en diferentes países alrededor del mundo (Baker, 2012; Rosenzweig, 1992; Sexton \& Hogan, 1992; Stevens \& Wedding, 2004). En lugar de un análisis histórico profundo, estos trabajos presentan descripciones que enfatizan momentos sobresalientes de la diseminación de la empresa psicológica a las condiciones locales de un país o de una región. La constatación de la propagación del conocimiento psicológico, centro de estas narraciones, más que conducir a análisis que señalen las dimensiones políticas, culturales y sociales involucradas en este proceso, aplaude la modernización alcanzada con la llegada de la psicología. Con estas miradas tradicionales se contribuye tanto a establecer unos límites para la disciplina, al señalar aspectos particulares que ocurren en cada ámbito a donde la psicología se expande, como a la construcción de un tipo peculiar de universalidad para el conocimiento psicológico, que tiene como base la celebración de su propia diseminación (Pulido-Martínez, 2017; Staeuble, 2006).

En vía contraria, como bien señala Staeuble (2006), ha empezado a constituirse una corriente de investigaciones que se aparta de esa forma apolítica de considerar la expansión de la psicología. Los trabajos presentados en los volúmenes editados por Blowers y Turtle (1987) y Carr y Schumaker (1996) se convierten en muestras de estudios pioneros que se han encargado de establecer las implicaciones que tiene la expansión de la psicología alrededor del mundo. Con las transformaciones que han ocurrido con el desarrollo de la "nueva" historia de la psicología (Brock, 2017), las propuestas conceptuales formuladas dentro de los estudios postcoloniales (Mahone \& Vaughan, 2007) y con el advenimiento de la psicología crítica y los estudios sociales de la ciencia (Jaraba-Barrios \& Mora-Gámez, 2010), ha sido posible empezar a examinar de manera distinta la constante expansión que ha tenido el conocimiento psicológico desde los países del Atlántico norte hacia el resto de Europa, América Latina, África y Asia (Brock, 2006; Lindtrum, 2016; Mahone \& Vaughan, 2007; Wu \& Wang, 2016). Al considerar el surgimiento y la diseminación de la psicología, los estudios en esta línea se apartan de visiones que suponen que las ideas psicológicas se dispersan alrededor del mundo gracias a la garantía de su cientificidad. En su lugar, se propone que los objetos psicológicos son construidos en la conjunción entre una o unas culturas, un momento específico, un espacio particular y un estado general del conocimiento. De este modo, se consigue dejar de lado el supuesto comúnmente aceptado de que la psicología es ahistórica y universal (Castel, 1984; Richards, 2002; Rose, 1996, 1999 ).

En otras palabras, los estudios que empiezan a observar las relaciones internacionales en las que está envuelto el conocimiento psicológico destacan que este siempre está espacializado, es decir, inmerso en relaciones locales y globales de poder (Molinari, 2004; Staeuble, 2004, 2005, 2006). Son, en este sentido, investigaciones que se podrían ubicar bajo el rótulo de geopolíticas, en la medida que se concentran en diferencias referidas a las relaciones internacionales en las cuales se constituye y disemina la psicología alrededor del mundo. Varias consecuencias vienen de la mano cuando se piensa la empresa psicológica como una cuestión universal con manifestaciones locales. La primera es que los objetos e historias oficiales de la psicología, que se presentan como mundiales, se tornan en lo que realmente son: versiones locales de lo que la disciplina es en un determinado momento (Brock, 2006). La segunda consecuencia ocurre al señalar que la psicología producida en los países que tradicionalmente han definido los objetos psicológicos puede dejar de considerarse como la manera cierta y, por tanto, mejor, de hacer psicología, lo cual trae como resultado que las demás regiones del planeta dejen de ser consideradas como aquellas que hacen aportes exóticos al conocimiento psicológico o bien como aquellas que se encargan, a través de procesos de adaptación, réplica y emulación, de confirmar la universalidad de las propuestas constituidas de antemano en las sociedades del Atlántico norte (Pulido-Martínez, 2017). La tercera consecuencia se refiere a la necesidad de que se ponga bajo examen la manera en que se apropia acríticamente la psicología en diferentes partes del mundo. Esto significa considerar como un asunto central que la expansión de la psicología se presenta entre aquellos que 
producen el conocimiento psicológico y aquellos que lo replican, apropian e hibridan, por tanto, se deben considerar las implicaciones que surgen de esta diferencia en términos históricos, culturales, políticos, económicos y sociales (Pulido Martínez, 2017). Y, por último, pero no menos relevante, la cuarta consecuencia señala que cuando se examina la geopolítica del conocimiento psicológico, se está ad portas de considerar, o mejor, en la obligación de proponer y de buscar, herramientas teóricas y metodológicas para realizar análisis globales, multisituados y simultáneos, entre lugares de producción y de consumo, que permitan mostrar la inserción de la psicología dentro de un orden internacional de la producción de conocimiento (Moghaddam, 1987; Pulido-Martínez, 2017; Pulido-Martínez, García-Álvarez, Carvajal-Marín \& González-Ortiz, 2013).

\section{Riesgos Psicosociales y Geopolítica del Conocimiento}

En este marco se sitúa el estudio de las tesis de pregrado y postgrado sobre los riesgos psicosociales en el trabajo que aquí se presenta. El análisis de las tesis llevadas a cabo con relación a los riesgos psicosociales que emergen del y en el trabajo no es casual; responde a la necesidad de considerar la geopolítica del conocimiento referida a un campo que se ha tornado central para las prácticas, intervenciones y explicaciones que circulan en relación con la psicología del trabajo y su campo de acción, la administración del mundo laboral. Las tesis con las cuales los alumnos cierran sus ciclos de formación se convierten en vehículos privilegiados para el estudio de la geopolítica de la psicología, en la medida en que se considera, por ejemplo, que la información allí consignada no solamente da cuenta de cómo localmente está el área de la cual hacen parte estos estudios (Molina Montoya, 2005), sino que también, a través de los análisis que se conducen, se ubican en conjunto esos trabajos investigativos dentro de un contexto geográfico específico (Rodríguez Medina, 2013).

En esta línea, la producción de conocimiento sobre los riesgos psicosociales en el trabajo tiene una posición privilegiada para adelantar análisis geopolíticos de la psicología por diversas razones. En primer lugar, alrededor de los riesgos psicosociales, se han creado instrumentos para su medición que traspasan las fronteras de sus países de origen, organizaciones internacionales promueven conceptos y estrategias para su control y se llevan a cabo adaptaciones de baterías y de cuestionarios particulares por parte de sectores oficiales, con la intención de establecer de manera obligatoria la medición de los riesgos psicosociales, como una forma para alcanzar un mejor gobierno de la fuerza laboral. Actualmente, por ejemplo, México, Chile y Colombia han instaurado instrumentos oficiales para la medición de riesgos psicosociales basados en propuestas desarrolladas en los centros productores de este conocimiento que son adaptados para ser aplicados a las muy variadas condiciones de trabajo de cada uno de estos países (Alvarado et al., 2012; Charria, Sarsosa \& Arenas, 2011; Colombia, Ministerio de la Protección Social \& Pontificia Universidad Javeriana, 2010; Moreno Jiménez, 2011). Esta situación, en la que se muestra la importancia alcanzada por los riesgos psicosociales en la regulación internacional del trabajo, ha sido objeto de alabanzas y celebraciones, mas no de mayores análisis críticos interesados en mostrar las implicaciones que a distintos niveles están involucrados en la expansión de la psicología.

En segundo lugar, los riesgos psicosociales en el trabajo tienen una relativamente reciente configuración como área de interés académica. Esta situación permite observar con facilidad cómo el campo ha sido fuertemente influenciado por agencias internacionales que promueven la regulación del trabajo. En efecto, los estudios muestran cómo la visión que considera al trabajo como riesgo, y los riesgos del trabajo, en términos psicosociales emergió en los años 70 y se consolidó en la década de los 80, cuando agencias como la Organización Internacional del Trabajo (OIT) y la Organización Mundial de la Salud (OMS) de manera conjunta publicaron una serie de investigaciones en donde se daban pasos para la circunscripción de esa relación entre lo psicosocial y el trabajo (Moreno Jiménez, 2011). En los años 90 el área se delimita en la literatura con lo que podría denominarse un canon incipiente, del cual se prescribe que puede ser utilizado alrededor del mundo para el manejo de estos riesgos (Kalimo \& Cooper, 1988; OIT, 1986; OIT \& OMS, 1984). En términos de las definiciones preferentes y ampliamente aceptadas acerca del concepto del riesgo psicosocial en el trabajo, se imponen aquellas realizadas en colaboración entre la OIT y la OMS en 1984 y 1986 (Forastieri, 2016; Leka \& Jain, 2010; OIT, 2016). Con ellas se hace referencia a las posibles influencias negativas que pueden tener sobre la salud del trabajador las condiciones laborales. Se trata de interacciones que se dan en el espacio y en las actividades propias del trabajo, en medio de relaciones organizacionales y sociales específicas, con sus consecuentes exigencias, necesidades y recursos propios. En términos generales y de muy distintas maneras, se considera cómo estas relaciones y actividades propias del trabajo interactúan con la cultura, capacidades, conocimientos y valores del trabajador, así como con sus percepciones y 
experiencias previas, de tal manera que le pueden producir daño o enfermedad, a nivel tanto físico como psicológico (emocional, cognitivo o comportamental) y/o social. En esta forma de definir los riesgos psicosociales se incluyen tanto las prescripciones que se dictan desde los estamentos internacionales como se consideran las particulares condiciones laborales en las que se desenvuelve el trabajo en los ámbitos locales. Vale anotar que en los últimos años los investigadores han formalizado una serie de modelos para definir, entender e intervenir los riesgos psicosociales en el trabajo, que se han convertido en las maneras corrientes de considerarlos. Estos modelos llevan consigo unas imágenes de la subjetividad psicologizada del trabajador, cuestión que recientemente ha sido descrita y criticada en estudios que realizan miradas generalizadas a la producción del campo (Allard-Poesi \& Hollet-Haudebert, 2012).

Finalmente, uno de los aspectos más importante para acercase al estudio de los riesgos psicosociales en el trabajo como un asunto de geopolítica del conocimiento, resulta de la particular relación que en la actualidad está teniendo este campo con la psicología del trabajo en su conjunto. A este respecto, en la literatura se ha explorado cómo la psicología del trabajo propuso una serie conceptos y estrategias para ser aplicados en relación con el empleo. La visión humanista que formuló la psicología imperó hasta los años 70 , con sus propuestas sobre la satisfacción laboral como centro de las intervenciones. A partir de esa década, gradualmente los enfoques sobre los riesgos psicosociales han colonizado las maneras de pensar e intervenir los problemas del trabajo, de forma tal que las estrategias tradicionales de la psicología industrial y organizacional, tales como la selección y la capacitación, son analizadas hoy en día a la luz de su relación con la prevención del estrés laboral (Pulido-Martínez \& Carvajal-Marín, 2016). De esta manera, las perspectivas que consideran los riesgos psicosociales en el trabajo están desplazando, en la práctica cotidiana de las organizaciones, a las propuestas tradicionales que han conformado la psicología. Así, alrededor de los riesgos psicosociales emergen por doquier nuevas formas para la administración de la fuerza de trabajo (PulidoMartínez \& Carvajal-Marín, 2016).

Ahora bien, estudiar las tesis sobre los riesgos psicosociales en el trabajo se convierte en una manera de acercarse en la práctica cotidiana a la forma en la cual se produce, o mejor, se hace tangible, la geopolítica de la psicología. Es decir, en estos estudios se establece un posible terreno sobre el que se asentará la futura práctica profesional de los graduados. En otras palabras, se estudia cómo en las tesis ocurre una dimensión de concreción de la práctica científica que resulta clave para entender cómo se espacializa la ciencia en "ciertos entornos, con ciertos recursos y bajo ciertas reglas" (Rodríguez Medina, 2013, p. 10). Las tesis, en la medida en que se supone producen algún aspecto nuevo del conocimiento, así como la apropiación particular de un campo por parte de un grupo de estudiantes, representan un camino adecuado para conocer cómo se constituye un área que favorece en la producción científica la construcción de lugares en donde se produce psicología y sitios en donde esta llega para su adaptación, réplica, emulación, apropiación e hibridación. Se puede, entonces, ver, a través del estudio de las tesis, la constitución de los centros y periferias del conocimiento psicológico (Biegel, 2013).

Las diferencias entre estos centros y periferias, o entre lugares de producción y de adaptación, ya han sido descritas por los investigadores para otros campos del conocimiento distintos a la psicología. Según Rodríguez Medina (2013), se presentan unos campos que están institucionalizados en un lugar geográficamente determinado y con el suficiente prestigio e insumos de todo tipo, y otros que entran en relación en términos de red, localizados en lugares marginales, o alrededor de los primeros, en donde se reciben y se apropian los planteamientos ya constituidos. Este tipo de situaciones da paso a que se establezcan, en donde se produce el conocimiento, relaciones consolidadas, compactas y sólidamente institucionalizadas y, en donde este se adapta, replica y apropia, unas relaciones fragmentarias y dispersas (Rodríguez Medina, 2013).

Hasta hace muy poco tiempo han empezado a aparecer estudios explícitamente destinados a examinar el potencial que tienen los recuentos estadísticos de la producción académica para auscultar asuntos relacionados con la geopolítica del conocimiento (Gómez-Morales \& Jaraba-Barrios, 2012,). Esta ausencia de estudios se relaciona con que la mayoría de las investigaciones de ese corte, si bien refinadas e indispensables para comparar los avances que ha mostrado la ciencia local frente a la ciencia global (Dávila Rodríguez et al., 2009; Naseer \& Mahmood, 2009), también han ignorado que, al concentrarse en análisis internos de un área del conocimiento, dejan de lado aspectos relacionados con las condiciones y relaciones particulares de producción y consumo del conocimiento en general (Sánchez-Tarragó, Bufrem \& dos Santos, 2015). Entonces, los estudios que revisan un campo en términos de recuentos de la producción han empezado a apoyarse en una serie de perspectivas conceptuales y metodológicas que buscan, a la manera de los estudios cualitativos documentales, hacer interpretaciones acerca de los datos en contexto (Alvarenga, 1998; Molina Montoya, 
2005). En este sentido, hay que considerar que, de por sí, no solo resulta relevante analizar las tesis en referencia a los avances que se pueden hacer en estas investigaciones o en términos de la formación de los futuros investigadores y practicantes de la disciplina, sino también resulta valioso traspasar estos límites.

\section{Diseño}

\section{Método}

Para acercase al examen de la geopolítica de la psicología en referencia a la producción hecha en las tesis que consideraron los riesgos psicosociales en el trabajo, el estudio partió de la constitución de un corpus documental base, sobre el cual se pudieron realizar análisis, tanto cuantitativos como cualitativos. Este corpus documental consideró los siguientes aspectos: el año de presentación de las tesis, la institución académica que las acogió y el programa específico al que estaban vinculadas, el título asignado a la investigación, el tipo de estudio realizado, los temas particulares considerados y el nivel académico al cual correspondían: pregrado o postgrado. También se establecieron las citas realizadas en cada tesis, así como el idioma en el cual estaban publicadas, las frecuencias de estas y el tipo de autor citado (institucional o investigador particular). Se siguieron las recomendaciones formuladas corrientemente para llevar a cabo investigaciones que buscan hacer un recuento e interpretación de la producción de conocimiento, tales como las propuestas por Jaraba-Barrios, Guerrero-Castro, Gómez-Morales y López-López (2011), así como por Barbosa Chacón, Barbosa Herrera y Rodríguez Villabona (2013).

\section{Muestra y Procedimiento}

Las universidades en las que reposaban las tesis fueron visitadas por estudiantes de psicología, previamente capacitados en las dimensiones del estudio, quienes actuaron como auxiliares para la recolección de la información directamente de los textos. Inicialmente, se ubicaron las tesis de pregrado y postgrado realizadas en diferentes facultades de las siguientes universidades: Nacional, de los Andes, del Rosario, Católica, El Bosque, Santo Tomás y la Pontificia Universidad Javeriana. Todas estas instituciones se hallan afiliadas a la Asociación Colombiana de Universidades.

Se tomó como criterio de inclusión que los textos estuvieran clasificados bajo las palabras clave riesgos psicosociales laborales y riesgos psicosociales en el trabajo. Se consideraron todos los campos disponibles para la búsqueda en los catálogos de las bibliotecas. Se hizo la revisión para cada uno de estos criterios y se eliminaron los títulos que estuvieran repetidos. En total, se consideraron 108 tesis en todas las dimensiones que compusieron el corpus documental. Hay que anotar que, por dificultades de diverso tipo, como la imposibilidad de acceder a algunos de los documentos en las instituciones, 14 tesis no quedaron reseñadas dentro del estudio. El intervalo de tiempo examinado estuvo determinado por las fechas de presentación del total de las referencias encontradas. Los años en los cuales fueron realizadas las tesis dan cuenta del interés creciente que en las últimas décadas ha tenido el área de los riesgos psicosociales asociados a diferentes ámbitos laborales, como una manera de entender los problemas que surgen allí.

\section{Análisis de Datos}

El corpus documental fue analizado a la luz de las recomendaciones sugeridas en la literatura para la fase heurística de los estudios que examinan conjuntos de producción en algún área del conocimiento (Barbosa Chacón et al., 2013; Jaraba-Barrios et al., 2011). Por tanto, se llevaron a cabo análisis descriptivos por medio de frecuencias y porcentajes en cada uno de los aspectos que fueron considerados en relación con las tesis de pregrado y postgrado. Siguiendo las prescripciones presentes en la literatura para efectos de lecturas de corte bibliométrico, se incluyeron los diferentes niveles de formación, por una parte, porque se tiene una visión de conjunto de los temas, los programas y las herramientas conceptuales y metodológicas que se han utilizado. Por otra parte, se puede reconocer en los datos globales de pregrado y postgrado que existen variaciones en la formación, más allá del foco y de los alcances particulares de cada uno de los estudios. El panorama general de las tesis de distintos niveles se elaboró bajo el supuesto de coherencia y continuidad conceptual y, por tanto, formativa, entre los niveles más avanzados y aquellos propuestos para llevar a cabo en el pregrado. De este modo, se arrojaron datos sobre los vínculos de la producción y circulación del conocimiento con procesos de réplica, adaptación, subordinación y universalización que ocurren gracias a y en razón de la expansión de la psicología. 


\section{Resultados}

En la Tabla 1 se muestra la distribución de las tesis relacionadas con riesgos psicosociales en el trabajo por cada una de las universidades que las albergaron. De este modo, se presenta un panorama de cuáles y con qué frecuencia las instituciones se han mostrado interesadas por un campo específico. Particularmente, para este estudio se encontró que el mayor número de tesis se condujo en la universidad El Bosque, con un 23,15\%, seguido de la Universidad Javeriana, con 18,52\%. Las universidades Católica, Santo Tomás, Rosario, de los Andes y Nacional sumaron el 50,93\%.

Tabla 1

Distribución de las Tesis en las Universidades Consideradas

\begin{tabular}{lcc}
\hline Universidad & Número de tesis & $\%$ \\
\hline del Rosario & 8 & 7,41 \\
Nacional & 12 & 11,11 \\
de los Andes & 13 & 12,04 \\
Santo Tomás & 14 & 12,96 \\
Católica & 16 & 14,82 \\
Javeriana & 20 & 18,52 \\
El Bosque & 25 & 23,15 \\
Total & 108 & 100,00 \\
\hline
\end{tabular}

El mayor número de tesis escritas correspondientes a cada institución y programa se presentó dentro de los postgrados en psicología de las universidades El Bosque y Católica. Esto puede sugerir la posibilidad de que se estén llevando a cabo los primeros pasos de un proceso de consolidación de líneas de investigación. Empero, no resultan menos relevantes los datos referidos a las facultades en donde se produce el conocimiento psicológico. Así, el $27,8 \%$ de los estudios lo componen tesis hechas en los programas de pregrado y postgrado de facultades diferentes a las de psicología (Tabla 2). Este porcentaje permite indicar no solamente los lugares específicos, la espacialización, en donde se hace la psicología; también muestra cómo los conceptos psicológicos, al unirse con las conceptualizaciones del riesgo como asunto psicosocial, traspasan las fronteras de la disciplina y van a presentarse en diferentes programas, entre los que se cuentan ingeniería, enfermería, administración de empresas y medicina. La distribución de los estudios en las diferentes facultades señala, por una parte, el interés interdisciplinar que despierta el campo de los estudios de riesgo psicosocial y, por otra, el desdibujamiento de los límites académicos del conocimiento psicológico que se produce cuando el riesgo se introduce en las conceptualizaciones para gobernar y, por tanto, intervenir el mundo laboral.

En concordancia con el desvanecimiento de las fronteras disciplinares de la psicología, reportado alrededor del mundo (Rose, 2008), la distribución que se presenta en la Tabla 2 en referencia a programas académicos en donde se lleva a cabo la investigación, resulta ser en su conjunto un indicador de que se está operando un cambio en términos de los agentes que hacen uso de la psicología. Estos agentes, como ya ha sido señalado para las sociedades del Atlántico norte, en donde se producen los objetos psicológicos, no son exclusivamente psicólogos, sino que, por el contrario, son muchos los profesionales y no profesionales de esta disciplina que hacen uso de este conocimiento (Rose, 1996). Cabe, entonces, acogerse al llamado que han hecho los investigadores para señalar que esta diversificación de usuarios es una de las manifestaciones que asume el proceso de psicologización que hoy en día recorre el mundo del trabajo, en el cual la disciplina psicológica coloniza otras profesiones y, por tanto, subordina, acalla o invisibiliza otras posibles maneras, locales y globales, de concebir al trabajo, a los trabajadores, al lugar de las condiciones objetivas de trabajo y a las organizaciones (Crespo \& Serrano Pascual, 2012; De Vos, 2012). 
Tabla 2

Tesis Distribuidas por el Tipo de Programa en Cada Universidad

\begin{tabular}{|c|c|c|c|}
\hline Universidad & Programa & Facultad & Total \\
\hline \multirow[t]{2}{*}{ El Bosque } & Postgrado & Psicología & 23 \\
\hline & Pregrado & Psicología & 2 \\
\hline \multirow[t]{2}{*}{ Católica } & Postgrado & Psicología & 4 \\
\hline & Pregrado & Psicología & 12 \\
\hline \multirow[t]{4}{*}{ Santo Tomás } & Pregrado & Psicología & 8 \\
\hline & Línea de investigación de estrés & Psicología & 2 \\
\hline & División ciencia de la salud & Psicología & 3 \\
\hline & Campo de salud y calidad de vida & Psicología & 1 \\
\hline \multirow[t]{4}{*}{ de los Andes } & Postgrado & Ingeniería Civil y Ambiental & 1 \\
\hline & Postgrado & Administración de Empresas & 1 \\
\hline & Postgrado & Psicología & 2 \\
\hline & Pregrado & Psicología & 9 \\
\hline \multirow[t]{3}{*}{ Nacional } & Postgrado & Enfermería & 10 \\
\hline & Postgrado & Ciencias Humanas & 1 \\
\hline & Pregrado & Ciencias Humanas & 1 \\
\hline \multirow[t]{6}{*}{ Javeriana } & Postgrado & Medicina & 1 \\
\hline & Pregrado & Psicología & 10 \\
\hline & Postgrado & Ingeniería & 1 \\
\hline & Pregrado & Ingeniería & 1 \\
\hline & Postgrado & Enfermería & 6 \\
\hline & Pregrado & Enfermería & 1 \\
\hline \multirow[t]{3}{*}{ del Rosario } & Postgrado & Psicología & 2 \\
\hline & Postgrado & Rehabilitación y Desarrollo Humano & 2 \\
\hline & Postgrado & Administración de Empresas & 4 \\
\hline Total & & & 108 \\
\hline
\end{tabular}

Si se consideran las maneras en que se distribuyen los estudios de tesis a lo largo de los años (Tabla 3), se denota que la distribución de los trabajos deja entrever la dispersión en que estos se encuentran.

En primera instancia, se presenta falta de continuidad en términos de frecuencia en la producción en las distintas universidades. Al mismo tiempo, se muestra un cierto conglomerado de estudios que ocurren alrededor de los postgrados, en donde se agrupan las tesis principalmente en las facultades de enfermería y de psicología de las universidades Javeriana, El Bosque y Nacional (Tabla 3). Sin embargo, valdría anotar que las tesis analizadas están dedicadas a la identificación y/o evaluación, mediante el uso de instrumentos diseñados para tal efecto, de los factores de riesgo en distintos ámbitos institucionales. Por tanto, más que producción de objetos psicológicos novedosos o autoctonizados, en la medida en estos estudios se han concentrado en aplicaciones de instrumentos a distintas poblaciones, pareciera estar repitiéndose lo que ha sido el fenómeno típico de la periferia, llamado por Blowers, Cheung y Ru (2009) como la emulación del conocimiento psicológico proveniente de los países del Atlántico norte.

Se observa en la Tabla 4 que para la elaboración de las tesis se tuvieron en cuenta un total de 4777 referencias. En promedio, en la Universidad Católica se consideraron 68 referencias por tesis; por el contrario, en la Universidad El Bosque el promedio únicamente ascendió a 31,08 referencias. Estas diferencias podrían estar señalando variaciones en los criterios que se establecen en las instituciones en relación con los requisitos que deben observarse en determinado momento para que una tesis se configure y acepte como adecuada para cerrar el ciclo de formación. 
Tabla 3

Distribución Anual de las Tesis por Universidad

\begin{tabular}{|c|c|c|c|c|c|c|c|}
\hline \multirow{2}{*}{ Año } & \multicolumn{7}{|c|}{ Universidad } \\
\hline & El Bosque & Católica & Santo Tomás & de los Andes & Nacional & Javeriana & del Rosario \\
\hline 1991 & & & & & & 1 & \\
\hline 1992 & & & & 1 & & & \\
\hline 1993 & & & & & & 1 & \\
\hline 1994 & & & & 2 & & 1 & \\
\hline 1995 & & & & & & & \\
\hline 1996 & & & & & & 2 & \\
\hline 1997 & & 2 & & & & 1 & \\
\hline 1998 & & 1 & & 1 & & & \\
\hline 1999 & & & 4 & & & & \\
\hline 2000 & & 2 & 4 & & & & 1 \\
\hline 2001 & & & & 1 & 1 & & 2 \\
\hline 2002 & & 1 & & & 1 & 1 & \\
\hline 2003 & & & 1 & & & & \\
\hline 2004 & & 1 & & 1 & & & \\
\hline 2005 & & 1 & & 1 & & & \\
\hline 2006 & & 1 & 1 & 1 & 2 & & 1 \\
\hline 2007 & & & & & & 1 & \\
\hline 2008 & 1 & 1 & & & & 1 & \\
\hline 2009 & 3 & 1 & & & & 2 & \\
\hline 2010 & 8 & & & 2 & & 2 & \\
\hline 2011 & 7 & & 1 & 1 & 2 & 1 & \\
\hline 2012 & 1 & 2 & 3 & & 1 & 3 & 1 \\
\hline 2013 & 4 & 2 & & 1 & 1 & 2 & 1 \\
\hline 2014 & & & & 1 & 4 & & 1 \\
\hline Sin año & 1 & 1 & & & & 1 & \\
\hline Total & 25 & 16 & 14 & 13 & 12 & 20 & 8 \\
\hline
\end{tabular}

Tabla 4

Promedio de Referencias por Tesis en Cada Universidad

\begin{tabular}{lcc}
\hline Universidad & $\begin{array}{c}\text { Total de } \\
\text { referencias }\end{array}$ & $\begin{array}{c}\text { Promedio de } \\
\text { referencias }\end{array}$ \\
\hline del Rosario & 277 & 34,63 \\
de los Andes & 547 & 42,08 \\
Santo Tomás & 665 & 47,50 \\
Nacional & 676 & 56,33 \\
Javeriana & 747 & 37,35 \\
El Bosque & 777 & 31,08 \\
Católica & 1088 & 68,00 \\
Total & 4777 & 44,23 \\
\hline
\end{tabular}

El número de referencias institucionales utilizadas ascendió a la suma de 677 (Tabla 5), mientras que las referencias hechas de autores específicos alcanzaron, por diferencia, la cifra de 4100, constituyéndose, de este modo, el 14,13\% para las primeras y el 85,87\% para las segundas. Así, independientemente de la dispersión que se hacía notar anteriormente en términos de la producción en las universidades, aparece un patrón aglutinador referido al papel que juegan las instituciones reguladoras del trabajo en este campo. La mayor frecuencia en términos de referencias institucionales corresponde al Ministerio de la Protección Social. A esta frecuencia pueden agregársele las demás referencias concernientes tanto al Ministerio del Trabajo como 
aquellas conjuntas entre el Ministerio del Trabajo y el Ministerio de Salud, puesto que son variaciones en las maneras de citar la misma entidad oficial. Cabe aclarar aquí que estas distintas denominaciones para las carteras de salud y trabajo han sido el resultado de los cambios en la administración del Estado, que, ya sea el caso, las han unido o separado. Se convierten, entonces, las 117 referencias del Ministerio del Trabajo en la cifra más frecuente de fuentes consultadas en las investigaciones llevadas a cabo en las tesis.

Vale también hacer énfasis en el número de las demás fuentes institucionales nacionales, en donde se cobijan aquellas referidas al Instituto de Seguros Sociales, la unión del Ministerio del Trabajo y la Universidad Javeriana, el Instituto Colombiano de Normas Técnicas y Certificación, el Ministerio de Salud, el Congreso de la República y el Departamento Administrativo Nacional de Estadística, las cuales alcanzan a sumar 140 referencias (Tabla 5). De este modo, el número total de referencias institucionales nacionales llega a la cifra de 257 (37,96\%), las que, en conjunto con las 420 referencias de entidades restantes (62,04\%), estarían señalando el lugar central que tienen los documentos, disposiciones, decretos y maneras de proceder que se generan a partir de estas entidades para la regulación del campo de los riesgos psicosociales y, por supuesto, para el tipo, las necesidades y los requerimientos que se pueden y deben alcanzar en los estudios que se adelanten en este campo. En esta línea de ideas, por consiguiente, los documentos oficiales contribuyen de manera central a determinar a la vez los límites de lo que puede y no puede considerarse como riesgos psicosociales, los debates que son pertinentes, así como los contenidos indispensables que deben tenerse en cuenta en la formación de los profesionales que vayan a ejercer. Estos resultados muestran, también, como en el campo de los riesgos psicosociales se constituye una estrecha y particular relación entre los ejercicios académicos que se realizan y la regulación del trabajo que se ejecuta por parte de las entidades oficiales que lo gobiernan.

No obstante, si estos datos señalan localizaciones e indican formas de apropiación del conocimiento psicológico que ocurren a través de entidades oficiales en países periféricos como Colombia, las fuentes utilizadas en las tesis, adicionalmente, revelan conexiones que se establecen con entidades reguladoras del trabajo al nivel internacional. Se observa, entonces, que las referencias utilizadas por los autores consideran también los documentos provenientes de instituciones internacionales y de entidades regulatorias de otros países diferentes de Colombia. La OIT y la OMS se referencian en 133 oportunidades (Tabla 5). Igualmente, documentos producidos por otras entidades de regulación regional distantes de Latinoamérica, como aquellos promulgados por la Fundación Europea para el Mejoramiento de las Condiciones de Vida y el Instituto Nacional de Seguridad e Higiene en el Trabajo de Barcelona, España, son tomadas en cuenta (6,06\%). Por las implicaciones ya señaladas en cuanto a apropiación y circulación del conocimiento psicológico en y entre los centros de producción del conocimiento y aquellos contextos en donde este llega para ser adaptado, replicado e hibridado, vale resaltar que el 14,71\% del total de las referencias está compuesto por aquellos documentos provenientes de entidades reguladoras nacionales e internacionales. No se requiere mayor suspicacia para reconocer el papel que estas organizaciones internacionales tienen, al convertirse en productoras y traductoras a los diferentes idiomas del conocimiento que se produce en los países en donde se generan los objetos psicológicos sobre el riesgo, para ponerlos al alcance de aquellos que hacen aplicaciones locales alrededor del mundo. Los marcos provistos por estas entidades delimitan las acciones particulares que se pueden llevar a cabo en los ámbitos organizacionales, al establecer qué puede ser considerado como riesgo psicosocial y cómo puede establecerse su presencia, al punto que, por ejemplo, la batería de riesgos psicosociales desarrollada entre el Ministerio del Trabajo y la Universidad Javeriana se ha convertido de uso obligatorio para todo tipo de empresas en Colombia.

En cuanto al recuento y distribución en términos de dónde se localiza la editorial que publicó las obras referenciadas y la vinculación institucional de los autores consultados, se presenta una nueva espacialización del conocimiento (Tabla 6). 
Tabla 5

Total Distribución de Citas Institucionales

\begin{tabular}{|c|c|c|c|c|c|c|c|c|}
\hline Institución & El Bosque & Católica & Javeriana & Santo Tomás & de los Andes & Nacional & del Rosario & Total \\
\hline Ministerio de la Protección Social & 49 & 4 & 6 & 7 & & 14 & 3 & 83 \\
\hline OIT & 14 & 6 & 6 & 11 & 1 & 15 & 9 & 62 \\
\hline Instituto de Seguros Sociales & 2 & 14 & 4 & 22 & 3 & 11 & & 56 \\
\hline OMS & 7 & 4 & 5 & 6 & 4 & 10 & 2 & 38 \\
\hline OIT y OMS & 7 & 9 & 4 & 5 & 2 & 2 & 4 & 33 \\
\hline $\begin{array}{l}\text { Instituto Nacional de Seguridad e Higiene en el Trabajo } \\
\text { (Barcelona, España) }\end{array}$ & 4 & 4 & 4 & 4 & & 12 & 3 & 31 \\
\hline Congreso de la República & 13 & 7 & 1 & 1 & & 3 & 4 & 29 \\
\hline Ministerio del Trabajo y Pontificia Universidad Javeriana & 14 & 3 & & 2 & & & & 19 \\
\hline Ministerio del Trabajo y Ministerio de Salud & 1 & 2 & 1 & 10 & & 3 & & 17 \\
\hline $\begin{array}{l}\text { Ministerio del Trabajo e Instituto Colombiano de Normas } \\
\text { Técnicas y Certificación }\end{array}$ & 7 & 3 & 3 & 1 & & & 3 & 17 \\
\hline Instituto Colombiano de Normas Técnicas y Certificación & 5 & 2 & & 2 & & 5 & & 14 \\
\hline Ministerio de Salud & & 5 & 3 & 1 & & 2 & & 11 \\
\hline Departamento Administrativo Nacional de Estadística & & & 3 & & 3 & 5 & & 11 \\
\hline $\begin{array}{l}\text { Fundación Europea para el Mejoramiento de las } \\
\text { Condiciones de Vida }\end{array}$ & 1 & 2 & & 1 & & 2 & 4 & 10 \\
\hline Otras instituciones & & & & & & & & 246 \\
\hline Total & & & & & & & & 677 \\
\hline
\end{tabular}


Tabla 6

Distribución de Referencias por País de la Editorial y por Vinculación Institucional del Autor

\begin{tabular}{|c|c|c|c|}
\hline Localización & Autor & Frecuencia & $\%$ \\
\hline \multirow[t]{9}{*}{ Colombia } & Villalobos (Universidad) & 59 & 15,57 \\
\hline & Sierra (Universidad) & 35 & 9,23 \\
\hline & Bossa (Oficial) & 14 & 3,69 \\
\hline & Rodríguez (Oficial) & 13 & 3,43 \\
\hline & Guerrero (Universidad) & 12 & 3,17 \\
\hline & Henao (Editorial) & 11 & 2,90 \\
\hline & Devia (Universidad) & 10 & 2,64 \\
\hline & Arenas (Editorial) & 16 & 4,22 \\
\hline & Subtotal & 170 & 44,85 \\
\hline \multirow[t]{3}{*}{ España } & Peiró & 35 & 9,23 \\
\hline & Gil-Monte & 23 & 6,07 \\
\hline & Subtotal & 58 & 15,30 \\
\hline \multirow[t]{6}{*}{ Unión Europea } & Lazarous y Folkman & 26 & 6,86 \\
\hline & Siegrits & 20 & 5,28 \\
\hline & Karaseck & 14 & 3,69 \\
\hline & Selye & 12 & 3,17 \\
\hline & Kalimo (Entidad internacional) & 10 & 2,64 \\
\hline & Subtotal & 82 & 21,64 \\
\hline \multirow[t]{3}{*}{ América } & Chiavenato & 15 & 3,96 \\
\hline & Muchinsky & 14 & 3,69 \\
\hline & Subtotal & 29 & 7,65 \\
\hline \multirow[t]{5}{*}{ Editorial internacional } & Mancilla (Editorial acceso abierto) & 15 & 3,96 \\
\hline & Hernández (Editorial internacional) & 13 & 3,43 \\
\hline & Ivancevich (Editorial internacional) & 12 & 3,17 \\
\hline & Subtotal & 40 & 10,56 \\
\hline & Total & 379 & 100,00 \\
\hline
\end{tabular}

Hay que anotar que, atendiendo a la manera en que se distribuyen las referencias cuando se tiene en cuenta el lugar en donde se ubica la entidad editorial que publicó el trabajo citado en las mismas (Tabla 6), emergen otros posibles canales por los cuales circula y se apropia el conocimiento psicológico, ahora articulado a la producción de autores colombianos. La red que hace posible la práctica psicológica con respecto a los riesgos psicosociales en el trabajo está también compuesta por los autores vinculados con universidades y entidades oficiales, así como por aquellos que escriben para editoriales específicas que seguramente cuentan con algún grado de especialización en el tema. El conjunto de autores conformado por Villalobos, Sierra, Guerrero y Devia muestra vinculación institucional con universidades locales, Bosa y Rodríguez tienen una filiación con entidades gubernamentales colombianas. Arenas y Henao se unen a ese conjunto para constituir el grupo de los autores nacionales más utilizados $(4,1 \%)$, es decir, con una frecuencia de más de 10 referencias dentro del total de las 108 tesis.

Esta red se complejiza aún más cuando se consideran los lazos internacionales de los autores que fueron referenciados. Peiró y Gil-Monte, dos autores españoles, se destacan de forma tal que se pone sobre el tapete el papel de la traducción de los conceptos e instrumentos psicológicos que se realiza en ese país europeo y su vinculación con los estudiantes en las universidades de Bogotá. Estos autores superan en frecuencia a la mayor parte de los autores colombianos (Tabla 6). La cifra de referencias de autores con vinculaciones internacionales se hace mayor cuando se consideran aquellas hechas de autores que publicaron sus trabajos a través de editoriales de acceso abierto e internacionales. Se completa, entonces, un total 88 referencias relacionadas con publicaciones hechas en el idioma español de circulación internacional.

En esta misma línea, cuando se examinan las frecuencias en las que se utilizan los trabajos de los autores que se reconocen como centrales para el campo de los riesgos psicosociales (Tabla 7), se tiene que Lazarus y 
Folkman fueron referenciados en 26 oportunidades y Siegrists, Karaseck, Selye y Kalimo, en su conjunto, solamente alcanzan el 1,7\% del total de las referencias. Este porcentaje señala el papel secundario que juegan aquellos a quienes se les reconoce como referentes centrales a nivel internacional en la producción de conceptos e instrumentos dentro del campo de los riesgos psicosociales (Allard-Poesi \& Hollet-Haudebert, 2012; Pulido Moreno, Puentes Suárez, Luney Cruz, López \& García Rubiano, 2015). El lugar no prioritario que ocupan las figuras del campo se puede relacionar con las dificultades de los estudiantes de la educación superior para el manejo del inglés como lingua franca. Por tanto, la traducción hecha en España permanece como mediadora entre los centros de producción y los lugares de aplicación y, de este modo, se convierte en elemento para la construcción de "la periferia de la periferia" del conocimiento. Empero, estas cifras resultan aún más indicadoras del lugar que tiene la traducción y las relaciones de centro-periferia en la circulación del conocimiento. Notablemente, los autores ubicados en los centros productores de conocimiento y destacados por la comunidad científica se ubican en su mayoría por debajo de las citas de traducciones hechas y apropiadas para España. El papel destacado de esas traducciones puede ser considerado como una muestra particular de interacción entre lo que Moghaddam (1987) ha llamado los mundos de la psicología. En este caso, en la medida en que la traducción llevada a cabo en España toma un lugar en las tesis, se produce una subordinación de la periferia (Colombia), a la ya de por sí periferia en términos de la producción del conocimiento psicológico (España), lo que podría denominarse, siguiendo el razonamiento propuesto por Moghaddam (1987), como un cuarto mundo de la psicología.

Tabla 7

Focalización del Estudio con Base en Títulos Propuestos en las Tesis

\begin{tabular}{lcc}
\hline \multicolumn{1}{c}{ Tema en el título } & Frecuencia & $\%$ \\
\hline Identificación de factores de riesgo en diversas poblaciones & 46 & 42,6 \\
Relación entre factores de riesgo y estrés & 16 & 14,8 \\
Relación entre factores de riesgo y otros tipos de variables & 15 & 13,9 \\
Programa de intervención & 13 & 12,0 \\
Diseños de cuestionarios para medir riesgos psicosociales & 5 & 4,6 \\
Estados del arte & 3 & 2,8 \\
Otros & 10 & 9,3 \\
Total & 108 & 99,9 \\
\hline
\end{tabular}

Al igual que otros estudios que han examinado la producción en lugares en donde no se producen los objetos psicológicos, sino que estos llegan como producto importado, cuando se consideran los temas de las tesis, a través de los títulos que les fueron asignados por los autores (Tabla 7), aparece un nuevo panorama que hace referencia al viaje de la psicología en términos más de emulación que de evolución de construcción de nuevos conceptos (Blowers et al., 2009).

En la medida en que los estudios, en su gran mayoría, tienen un corte descriptivo (Tabla 8), se puede afirmar que se limitan a utilizar los conceptos establecidos en la literatura, a través de ponerlos a prueba en poblaciones en donde no habían sido aplicados, para contribuir a hacerlos universales. El enfoque eminentemente práctico y técnico que tiene el campo en términos de los estudios analizados es una de las formas, o mejor, de los facilitadores que allanan los caminos por donde viaja la psicología alrededor del mundo. Se denota aquí cómo ocurre una apropiación de las propuestas para el manejo de los riesgos psicosociales que, más que hacer aportes conceptuales, permite hacer contribuciones en pos de la confirmación de los resultados que ya se han alcanzado en los lugares en donde se producen los objetos psicológicos (Pulido-Martínez, 2018). 
Tabla 8

Diseños de las Tesis Estudiadas

\begin{tabular}{lcl}
\hline \multicolumn{1}{c}{ Tipo de investigación/diseño } & Frecuencia & $\%$ \\
\hline Epidemiológico descriptivo & 1 & 0,93 \\
Tecnológico & 1 & 0,93 \\
Psicométrico & 1 & 0,93 \\
Ex post facto & 1 & 0,93 \\
Longitudinal prospectivo de panel & 1 & 0,93 \\
Estudio de caso & 2 & 1,85 \\
No experimental & 4 & 3,70 \\
Revisión documental & 9 & 8,33 \\
Estudios descriptivos & 63 & 58,33 \\
No especificado & 25 & 23,15 \\
Total & 108 & 100,01 \\
\hline
\end{tabular}

Basta agregar que el tipo de psicología que utiliza los cuestionarios, como ha sido señalado en los estudios históricos, se diseñó para transportar fácilmente a la psicología a diferentes lugares del planeta (Linstrum, 2016). Por tanto, este tipo de estudios que usa de forma regular los instrumentos, ahora adaptados para su uso en diferentes poblaciones, guarda una estrecha relación con las estrategias a través de las cuales se construye y perpetúa el lugar periférico, o de asimetría de poder, en cuanto a la geopolítica del conocimiento psicológico. Como ha sido bien descrito por la investigación en los estudios sociales e históricos de la ciencia, la emulación surge de las aplicaciones y adaptaciones de la psicología, en donde el caso que se localiza en la periferia puede en la mejor de las situaciones hacer una contribución exótica, única o particular, a la construcción del conocimiento universal (Gorbach \& López Beltrán, 2008), pero tiene muy limitadas las posibilidades de cuestionar, reformar o reformular los objetos psicológicos producidos en las sociedades del Atlántico norte (Pulido Martínez, 2017).

Esto no quiere decir que los estudios de tesis tengan un lugar poco importante. Por el contrario, periféricos como son dentro de la periferia, tienen un lugar fundamental en términos de la práctica psicológica, puesto que ellos constituyen un primer contacto independiente de los estudiantes con las maneras de hacer investigación. Dicho de otro modo, los estudiantes, al operar como investigadores, replican las formas en las cuales se establecen unos límites dentro de un campo, con los objetos y preguntas que son valiosos de indagar, así como, también, apropian la geopolítica del conocimiento que se traspasa en la misma ejecución de la investigación que se lleva a cabo en estos estudios. Todavía falta añadir que en la medida en que se hacen ejercicios investigativos, por pequeños que sean, los trabajadores objeto de estos estudios también entran en contacto con los conceptos y prácticas psicológicas y, en la medida en que ellos aportan información sobre su trabajo, sus relaciones y sus actividades, al mismo tiempo ocurre el fenómeno reflexivo propio de la psicología, que consiste en que cuando se realizan estudios psicológicos, estos contribuyen a la producción de una subjetividad en el trabajo que contemporáneamente se caracteriza por ser psicologizada, es decir, individualizada y en riesgo de enfermar por cuestiones psicosociales (Pulido-Martínez et al., 2013).

\section{Conclusiones}

El análisis de la producción de las tesis sobre los riesgos psicosociales puede ser fácilmente complementario del tipo de las investigaciones en las que se señala que, si bien la pregunta por el viaje de la psicología alrededor del mundo resulta pertinente, no se debe olvidar que este conocimiento y sus prácticas derivadas llegan a un terreno previamente arado (Pulido-Martínez et al., 2013). Aquí se ha señalado cómo las tesis hacen una contribución al sustrato de prácticas y conocimientos sobre los cuales llega, se apropia, cimienta y expande la psicología en relación con el riesgo psicosocial. Las condiciones locales en las cuales se recibe el conocimiento psicológico resultan sumamente importantes para entender cómo se universaliza la psicología. Es en este sentido que se ha descrito como en esos estudios se permea la geopolítica de la psicología, que se manifiesta en una compleja red de relaciones de poder y conocimiento.

Para que la psicología del trabajo se haga posible a través de las tesis, al igual que como ha sido establecido en la literatura para todas las ramas de la disciplina (Rose, 1996), no solamente se requiere un 
estatus de cientificidad; se precisa de toda una trama compuesta por editoriales, universidades, traductores, profesores, investigadores particulares, entidades internacionales, nacionales y locales encargadas de la regulación del trabajo y, por supuesto, estudiantes. Esta es la constelación de relaciones por donde circula la psicología que, en palabras de Castro-Gómez (2007), pueden ser descritas como cadenas de poder y conocimiento sumamente "enredadas", que entran en contacto en las tesis, dejando entrever formas en las cuales se expresa la geopolítica de la psicología.

Acercarse a la forma que asume la puesta en escena de la psicología (Rose, 1996) en localizaciones específicas, muestra la pertinencia de superar los límites que se autoimponen los estudios que se encargan de examinar a la psicología cuando se restringen a ver este conocimiento como una cuestión nacional. El examen de la trama de relaciones internacionales, nacionales, institucionales y personales que hace posible el conocimiento psicológico, es una cuestión central para desnaturalizar el lugar que tiene y las operaciones que cumple en las sociedades localizadas alrededor de aquellas en donde se producen los objetos psicológicos.

En este sentido, para el análisis de esta trama se debe tener en cuenta la complementariedad del proceso de producción y de apropiación que ocurre entre la periferia de la periferia y los centros productores y reguladores, tanto del conocimiento psicológico como del trabajo, pues es en esta articulación en donde se compone un terreno para que la psicología fluya con facilidad y no se le cuestione. En la complementariedad señalada se constituyen diferentes espacializaciones que mantienen conexiones distantes e insospechadas (Marcus, 1995), por medio de las cuales la psicología se hace realmente universal. Es, entonces, una conjunción de cadenas de poder, nacionales e internacionales, que le da paso al fenómeno de la psicologización como geopolítica que cada día cubre en mayor medida a los diferentes ámbitos laborales alrededor del mundo. Por supuesto, en el estudio que aquí se reporta estas cadenas apenas se vislumbran.

Por medio de la investigación se ha buscado hacer una contribución, al poner en cuestión el aparente lugar apolítico con el que se presenta el conocimiento psicológico, a través de una práctica que se presenta tan familiar y obvia como la elaboración de una tesis. Se debe señalar, entonces, que los resultados de este estudio hacen un aporte que contribuye a la incipiente discusión sobre la geopolítica del conocimiento; sin embargo, este aporte es un tanto modesto. Se circunscribe, y he aquí una limitación importante del estudio, a la producción hecha en la ciudad de Bogotá. Más que dar respuestas amplias, con este estudio se han abierto nuevos interrogantes acerca de la geopolítica de la psicología en su relación con los riesgos psicosociales y, quizá por extensión, se ha propuesto la urgencia de estudiar diversos aspectos que están involucrados, no solamente en la expansión de la psicología del trabajo, sino de la empresa psicológica misma.

También hay que decir que los datos y análisis aquí provistos, descriptivos como son, señalan que habrá que llevar a cabo futuros estudios que profundicen en los contenidos de las tesis y en la forma misma en que se lleva a cabo el proceso de su realización, con miras a entender en mayor medida como tiene lugar la articulación entre las prácticas y discursos psicológicos y la psicologización política del trabajo (Crespo Suárez $\&$ Serrano Pascual, 2011). Adicionalmente, resulta también pertinente establecer en futuras investigaciones las relaciones de continuidad y complementariedad que tienen lugar en otros espacios académicos periféricos para, de este modo, contribuir a comprender cómo la geopolítica de la psicología muestra, en sus diferencias, un carácter simultáneo, es decir, globalizante.

\section{Referencias}

Allard-Poesi, F. \& Hollet-Haudebert, S. (2012). La construction du subject souffrant au travail au travers des instruments scientiques de mesure [La construcción del sujeto que sufre en el trabajo, a través de los instrumentos scientíficos de medida]. @GRH, 4(5), 4574. https://doi.org/10.3917/grh.124.0045

Alvarado, R., Pérez-Franco, J., Saavedra, N., Fuentealba, C., Alarcón, A., Marchetti, N. \& Aranda, W. (2012). Validación de un cuestionario para evaluar riesgos psicosociales en el ambiente laboral en Chile. Revista Médica de Chile, 140, 1154-1163. https://doi.org/10.4067/S0034-98872012000900008

Alvarenga, L. (1998). Biliometria e arqueologia do saber de Michel Focault - traços de identidade teórico-metodológica [Bibliometría y arqueología del saber de Michel Foucault: los rasgos de la identidad teórico-metodológica]. Ciência da Informaçao (online), 27(3). https://doi.org/10.18225/ci.inf..v27i3.778

Baker, D. B. (Ed.) (2012). The Oxford handbook of the history of psychology: Global perspectives. Oxford, Reino Unido: Oxford University Press. https://doi.org/10.1093/oxfordhb/9780195366556.001.0001

Barbosa Chacón, J. W., Barbosa Herrera, J. C. \& Rodríguez Villabona, M. (2013). Revisión y análisis documental para estado del arte: una propuesta metodológica desde el contexto de la sistematización de experiencias educativas. Investigación Bibliotecológica, 27(61), 83-105. https://doi.org/10.1016/S0187-358X(13)72555-3

Beigel, F. (2013). Centros y periferias en la circulación internacional del conocimiento. Nueva Sociedad, 245, 110-123. Extraído de http://nuso.org/media/articles/downloads/3944_1.pdf 
Blowers, G., Cheung, B. T. \& Ru, H. (2009). Emulation vs. indigenization in the reception of Western psychology in Republican China: An analysis of the content of Chinese psychology journals (1922-1937). Journal of the History of the Behavioral Sciences, 45, 21-33. https://doi.org/10.1002/jhbs.20347

Blowers, G. H. \& Turtle, A. M. (Eds.) (1987). Psychology moving East: The status of Western psychology in Asia and Oceania. Sydney, Australia: Sydney University Press.

Brock, A. C. (Ed.) (2006). Internationalizing the history of psychology. New York, NY: New York University Press.

Brock, A. C. (2017). The new history of psychology: Some (different) answers to Lovett's five questions. History of Psychology, 20, 195217. https://doi.org/10.1037/hop0000036

Carr, S. C. \& Schumaker, J. F. (Eds.) (1996). Psychology and the developing world. Westport, CT: Praeger.

Castaño, D. A. \& Sánchez, G. (1978). Problemas de la importación tecnológica psicolaboral en los países en desarrollo. Revista Latinoamericana de Psicología, 10, 71-82. Extraído de http://www.redalyc.org/pdf/805/80511107.pdf

Castel, R. (1981/1984). La gestion de los riesgos: de la anti-psiquiatría al post-análisis (N. Pérez, Trad.; Título original: La gestion des risques). Barcelona, España: Anagrama.

Castro-Gómez, S. (2007). Michel Foucault y la colonialidad del poder. Tabula Rasa, 6, 153-172. https://doi.org/10.25058/20112742.290

Charria, V. H., Sarsosa, K. V. \& Arenas, F. (2011). Factores de riesgo psicosocial laboral: métodos e instrumentos de evaluación. Revista Facultad Nacional de Salud Pública, 29, 380-391. Extraído de http://www.redalyc.org/pdf/120/12021522004.pdf

Colombia, Ministerio de la Protección Social \& Pontificia Universidad Javeriana (2010). Batería de instrumentos para la evaluación de factores de riesgo psicosocial. Bogotá, Colombia: Autor. Extraído de http://fondoriesgoslaborales.gov.co/documents/Publicaciones/Estudios/Bateriariesgo-psicosocial-1.pdf

Crespo, E. \& Serrano Pascual, M. A. (2012). La psicologización del trabajo: la desregulación del trabajo y el gobierno de las voluntades. Teoría y Crítica de la Psicología, 2, 33-48. Extraído de http://teocripsi.com/ojs/index.php/TCP/article/view/82/68

Crespo Suárez, E. \& Serrano Pascual, A. (2011). Regulacion del trabajo y el gobierno de la subjetivdad: la psicologización política del trabajo. En A. Ovejero \& J. Ramos (Coords.), Psicología social crítica (pp. 246-263). Madrid, España: Biblioteca Nueva.

Danziger, K. (1997). Naming the mind: How psychology found its language. Thousand Oaks, CA: SAGE.

Dávila Rodríguez, M., Guzmán Sáenz, R., Macareno Arroyo, H., Piñeres Herera, D., de la Rosa Barranco, D. \& Caballero-Uribe, C. V. (2009). Bibliometría: conceptos y utilidades para el estudio médico y la formación profesional. Salud Uninorte, 25, 319-330. Extraído de http://www.redalyc.org/articulo.oa?id=81712365011

De Vos, J. (2012). Psychologisation in times of globalisation. London, Reino Unido: Routledge. https://doi.org/10.1080/14780887.2012.717837

Forastieri, V. (2016). Prevención de riesgos psicosociales y estrés laboral. Boletin Internacional de Investigacion Sindical, 8, 11-37. Extraído de http://www.ilo.org/wcmsp5/groups/public/---ed_dialogue/---actrav/documents/publication/wcms_553931.pdf

Gómez-Morales, Y. J. \& Jaraba-Barrios, B. (2012, Junio). La reconstrucción bibliométrica del pasado: un ensayo sobre la internacionalización de la ciencia. Ponencia presentada en las IX Jornadas Latinoamericanas de Estudios Sociales de la Ciencia y la Tecnología, Ciudad de México, México. https://doi.org/10.13140/2.1.2273.7925

Gorbach, F. \& López-Beltrán, C. S. (Eds.) (2008). Saberes locales. Ensayos sobre historia de la ciencia en América Latina. Zamora, México: El Colegio de Michoacán.

Herman, E. (1995). The romance of American psychology: Political culture in the age of experts. Berkeley, CA: University of California Press.

Jaraba-Barrios, B., Guerrero-Castro, J., Gómez-Morales, Y. J. \& López-López, W. (2011). Bibliometría e historia de las prácticas académicas locales: un esbozo a partir del caso de la psicología en Colombia. Avances en Psicología Latinoamericana, 29, 354-369. Extraído de https://revistas.urosario.edu.co/index.php/apl/article/view/2008

Jaraba-Barrios, B. \& Mora-Gámez, F. (2010). Reconstruyendo el objeto de la crítica: sobre las posibles confluencias entre psicología crítica y estudios sociales de la ciencia y la tecnología. Revista Colombiana de Psicología, 19, 225-239.Extraído de https://revistas.unal.edu.co/index.php/psicologia/article/view/12991

Jiménez, E. (2004). Análisis bibliométrico de tesis de pregrado de estudiantes venezolanos en el área de educación: 1990-1999. Extraído de https://rieoei.org/historico/deloslectores/623Jimenez.PDF

Kalimo, R., El-Batawi, M. A. \& Cooper, C. L. (Comps.) (1988). Los factores psicosociales en el trabajo y su relación con la salud. Genève, Suiza: Organización Mundial de la Salud. Extraído de http://apps.who.int/iris/bitstream/handle/10665/37881/9243561022_spa.pdf;isessionid=ECC8088FAD077D86C3F762D501B525EF?sequence=1

Leka, S. \& Jain, A. (2010). Health impact of psychosocial hazards at work: An overview. Genève, Suiza: World Health Organization. Extraído de http://apps.who.int/iris/bitstream/10665/44428/1/9789241500272_eng.pdf

Linstrum, E. (2016). Ruling minds: Psychology in the British Empire. Cambridge, MA: Harvard Universtiy Press. https://doi.org/10.1002/jhbs.21796

Mahone, S. \& Vaughan, M. (Eds.) (2007). Psychiatry and empire. New York, NY: Palgrave Macmillan. https://doi.org/10.1057/9780230593244

Marcus, G. E. (1995). Ethnography in/of the world system: The emergence of multi-sited ethnography. Annual Review of Anthropology, 24, 95-117. https://doi.org/10.1146/annurev.an.24.100195.000523

Moghaddam, F. M. (1987). Psychology in the three worlds: As reflected by the crisis in social psychology and the move toward indigenous third-world psychology. American Psychologist, 42, 912-920. https://doi.org/10.1037/0003-066X.42.10.912

Molina Montoya, N. P. (2005). ¿Qué es el estado del arte? Ciencia \& Tecnología para la Salud Visual y Ocular, 5, 73-75. https://doi.org/10.19052/sv.1666

Molinari, J. M. (2004). Hacia una nueva geopolítica de la psicología: prospectiva, conceptos y tendencias. Acta Psiquiátrica y Psicológica de América Latina, 50, 25-33.

Moreno Jiménez, B. (2011). Factores y riesgos laborales psicosociales: conceptualización, historia y cambios actuales. Medicina y Seguridad en el Trabajo, 57(Suplemento 1), 4-19. https://doi.org/10.4321/S0465-546X2011000500002

Naseer, M. M. \& Mahmood, K. (2009). Use of bibliometrics in LIS research. Libres, 19(2), 1-11. Extraído de https://cpb-use1.wpmucdn.com/blogs.ntu.edu.sg/dist/8/644/files/2014/06/Vol19_I2_Naseer_Mahmood_Ess_Op_909.pdf

Organización Internacional del Trabajo (1986). Factores psicosociales en el trabajo: reconocimiento y control. Genève, Suiza: Autor.

Organización Internacional del Trabajo (2016). Estrés en el trabajo: un reto colectivo. Turín, Italia: Autor.

Organización Internacional del Trabajo \& Organización Mundial de la Salud (1984). Factores psicosociales en el trabajo: naturaleza, inicidencia y prevención. Genève, Suiza: Autores.

Pulido Martínez, H. C. (2017). De la relación entre la psicología y el trabajo a la luz de sus relaciones coloniales. Estudos de Psicologia, 34, 5-14. https://doi.org/10.1590/1982-02752017000100002 
Pulido-Martínez, H. C. (2018). De la expansión internacional de la psicología a la simultaneidad de procesos: o de la investigación sobre la subjetividad en el trabajo. En D. Pavón-Cuellar (Coord.), Capitalismo y psicología crítica en Latinoamérica: del sometimiento neocolonial a la emancipación de subjetividades emergentes (pp. 243-264). México DF, México: Kanankil.

Pulido-Martínez, H. C. \& Carvajal-Marín, L. M. (2016). Patologización y despatologización de la ficción del "trabajador libre" en términos del riesgo psico-laboral. En A. A. Leal Ferreira, A. Molas \& J. Carrasco (Orgs.), Psicologia, tecnologia e sociedade: controvérsias metodológicas e conceituais para uma análise das práticas de subjetivação [Psicología, tecnología y sociedad: controversias metodológicas y conceptuales para un análisis de las prácticas de subjetivación] ( pp. 239-258). Rio de Janeiro, Brasil: NAU.

Pulido-Martínez, H. C., García Álvarez, C. M., Carvajal-Marín, L. M. \& González Ortiz, I. D. (2013). Entre bendiciones y perjuicios. Mapeando el lugar y las operaciones de la psicología en relación con el trabajo en el sector de la salud en Bogotá. En O. Restrepo Forero (Ed.), Proyecto ensamblado en Colombia. Tomo 1: Ensamblando estados (pp. 355-370). Bogotá, Colombia: Universidad Nacional de Colombia.

Pulido Moreno, N., Puentes Suárez, A., Luney Cruz, Z., López, D. R. \& García Rubiano, M. (2015). Análisis bibliométrico de la producción científica sobre riesgo psicosocial laboral, publicada entre 2000 y 2010. Diversitas, 11(1), 147-161. https://doi.org/10.15332/s17949998.2015.0001.10

Richards, G. (2002). The psychology of psychology: A historicaly grounded sketch. Theory \& Psychology, 12, 7-36. https://doi.org/10.1177/0959354302121002

Rodríguez Medina, L. (2013). Objetos subordinantes: la tecnología epistémica para producir centros y periferias. Revista Mexicana de Sociología, 75, 7-28. https://doi.org/10.22201/iis.01882503p.2013.1.35113

Rose, N. (1996). Inventing our selves: Psychology, power and personhood. Cambridge, Reino Unido: Cambridge University Press.

Rose, N. (1999). Governing the soul: The shaping of the private self (2a ed.). London, Reino Unido: Free Association Books.

Rose, N. (2008). Psicologia como uma ciência social [Psicología como una ciencia social]. Psicologia \& Sociedade, 20, 155-164. https://doi.org/10.1590/S0102-71822008000200002

Rosenzweig, M. R. (1992). Psychological science around the world. American Psychologist, 47, 718-722. https://doi.org/10.1037/0003066X.47.6.718

Sánchez-Tarragó, N., Bufrem, L. S. \& dos Santos, R. N. M. (2015). La producción científica latinoamericana desde una mirada poscolonial. Tendências da Pesquisa Brasileira em Ciência da Informação, 8(2), 182-202. Extraído de http://eprints.rclis.org/32986/1/2015produccion\%20cientifica\%20mirada\%20poscolonial.pdf

Sexton, V. S. \& Hogan, J. D. (Eds.) (1992). International psychology: Views from around the world. Lincoln, NE: University of Nebraska Press.

Staeuble, I. (2004). De-centering Western perspectives: Psychology and the disciplinary order in the first and third world. En A. C. Brock., A. Louw \& W. van Hoorn (Eds.), Rediscovering the history of psychology: Essays inspired by the work of Kurt Danziger (pp. 183-205). New York, NY: Kluwer Academic.

Staeuble, I. (2005). The international expansion of psychology: Cultural imperialism or chances for alternative cultures of knowledge? En A. Gülerce, A. Hofmeister, I. Staeuble, G. Saunders \& J. Kay, Contemporary theorizing in psychology: Global perspectives (pp. 88-96). Ontario, Canadá: Captus Press.

Staeuble, I. (2006). Psychology in the Eurocentric order of the social sciences: Colonial constitution, cultural imperialist expansion, postcolonial critique. En A. C. Brock (Ed.), Internationalizing the history of psychology (pp. 183-207). New York, NY: New York University Press.

Stevens, M. J. \& Wedding, D. (Eds.) (2004). Handbook of international psychology. New York, NY: Brunner/Routledge.

Wu, H. Y. -J. \& Wang, W. -J. (2016). Making and mapping psy sciences in East and Southeast Asia. East Asian Science, Technology and Society, 10, 109-120. https://doi.org/10.1215/18752160-3494245

Fecha de recepción: Abril de 2017.

Fecha de aceptación: Agosto de 2018. 